\title{
Melhorando Quadros de Horário de Escolas através de Caminhos Mínimos ${ }^{1}$
}

M.J.F. SOUZA², Departamento de Computação, Instituto de Ciências Exatas e Biológicas, Universidade Federal de Ouro Preto, 35400-000 Ouro Preto, MG, Brasil

N. MACULAN, Programa de Engenharia de Sistemas e Computação/COPPE, Universidade Federal do Rio de Janeiro, Cx.P. 68511, 21945-970 Rio de Janeiro, RJ, Brasil

L.S. OCHI, Departamento de Ciência da Computação, Universidade Federal Fluminense, Rua Passo da Pátria, 156, 24210-240 Niterói, RJ, Brasil.

Resumo. Um dos problemas básicos na elaboração de horários de aula de uma escola é o de compactar a agenda dos professores tanto quanto possível, satisfazendo a um conjunto de restrições de vários tipos. Trata-se de um problema NP-difícil e, assim, justifica-se a utilização de técnicas heurísticas para resolvê-lo. Apresenta-se uma técnica de busca local que, a partir de uma solução viável inicial, gera soluções melhores através da deteç̧ão de ciclos de custo negativo nos grafos associados aos quadros de horário de cada uma das turmas.

\section{Introdução}

A elaboração manual de horários de aula de uma escola é uma tarefa árdua. Um conjunto de restrições pedagógicas, administrativas e mesmo pessoais precisa ser satisfeito. Quanto muito, consegue-se uma solução a qual pode ser insatisfatória com relação a diversos aspectos. Por exemplo, um professor pode ficar insatisfeito se houver muitos buracos em sua programação semanal de ensino.

Esforços no sentido de automatizar a elaboração de quadros de horário tem sido despendidos [7]. Contudo, trata-se de um problema de difícil generalização. Em virtude da diversidade de regimes educacionais e das características de cada escola, os sistemas são desenvolvidos para atender, em geral, a uma instituição específica. Por outro lado, encontrar um quadro viável de horários é um problema NP-Completo [5], limitando o uso de técnicas exatas.

Entre as técnicas recentes utilizadas para resolver o problema, destacam-se as metaheurísticas: Busca Tabu [8, 4], Simulated Annealing [1], Algorítmos Genéticos [3], etc. Uma deficiência dessas aplicações é que não se explora de forma adequada

\footnotetext{
${ }^{1}$ Trabalho parcialmente financiado pelo PICDT/CAPES.

${ }^{2}$ Doutorando do Programa de Engenharia de Sistemas e Computação, COPPE/UFRJ.
} 
o fato de uma solução viável ter sido gerada (Em geral, as iterações prosseguem com o mesmo mecanismo de busca).

Apresentamos uma técnica de busca local que, baseada em movimentos dos horários das aulas das turmas, gera, a partir de uma solução viável inicial, soluções melhores através da detecção de ciclos de custo negativo nos grafos associados aos quadros de horário de cada uma das turmas. Essa técnica pode ser facilmente incorporada a essas metaheurísticas, de forma a melhorar uma solução viável obtida.

\section{Formulação do Problema}

O Problema do Horário de Escolas abordado consiste de um conjunto de $m$ professores, $n$ turmas, $s$ matérias e $p$ horários semanais reservados para a realização das aulas. Os $p$ horários semanais são distribuídos em $d$ dias da semana de $h$ horários diários realizados em um único turno, isto é, $p=d * h$. As turmas, as quais estão sempre disponíveis, são conjuntos disjuntos de estudantes que cursam as mesmas matérias. A cada matéria de uma turma está associado um único professor, previamente fixado. Além disso, a carga horária de cada turma é exatamente $p$.

O PHE considerado pode ser formulado como segue: Compactar a agenda dos professores, tanto quanto possível, satisfazendo às seguintes restrições:

(a) um professor não pode ministrar aula para mais de uma turma ao mesmo tempo;

(b) uma turma não pode ter aula com mais de um professor em um mesmo horário;

(c) cada professor $i$ tem que cumprir sua carga horária semanal $r_{i j}$ para cada turma $j$;

(d) um professor não pode ser alocado a um horário no qual não esteja disponível;

(e) uma turma não pode ter mais de dois horários de aula por dia com um mesmo professor;

(f) para um dado conjunto de matérias, as aulas devem obedecer a uma certa configuração semanal. Por exemplo, em uma matéria de 3 horas-aula semanais, a configuração a ser atendida pode ser 2-1, isto é, uma aula dupla (ministrada em dois horários consecutivos) e outra ministrada em um único horário, não necessariamente nessa ordem.

Por compactar a agenda dos professores, entende-se minimizar o número de buracos $b_{i}$ (horários ociosos entre dois horários de aula de um mesmo turno) na agenda de cada professor, bem como o número de dias $v_{i}$ em que cada professor está envolvido em alguma atividade. Mais precisamente, a função objetivo fo é calculada com base na expressão:

$$
f_{o}=\sum_{i=1}^{m} f_{o_{i}}=\sum_{i=1}^{m}\left(\alpha_{i} * b_{i}+\beta_{i} * v_{i}\right),
$$

onde $\alpha_{i}$ e $\beta_{i}$ são pesos e refletem a importância relativa de $b_{i}$ e $v_{i}$, respectivamente, na agenda de cada professor $i$. 


\section{Descrição do Procedimento}

Dado um quadro $Q$ de horários de professores, define-se o grafo da turma $j$ por $G_{j}=\left(V_{j}, A_{j}\right)$, onde $V_{j}$ é o conjunto dos horários reservados para a turma $j$ e $A_{j}$ é o conjunto dos arcos orientados definido conforme a seguir:

$A_{j}=\{(k, \bar{k})$ : a aula da turma $j$ ministrada no horário $k$ pode ser ministrada também no horário $\bar{k}\}$

Da construção do grafo $G_{j}$ deduz-se que para um arco orientado $(k, \bar{k})$ pertencer ao conjunto $A_{j}$ as seguintes condições precisam ser atendidas:

(a) o professor do horário $k$ precisa estar disponível no horário $\bar{k}$;

(b) as restrições relativas à aula do horário $k$ precisam estar, também, satisfeitas no horário $\bar{k}$.

A cada arco $(k, \bar{k}) \in G_{j}$ associa-se um custo $\Delta f o_{i}(k, \bar{k})$, o qual representa a variação do custo de se transferir o professor $i$ do horário $k$ para o horário $\bar{k}$. Esse custo é obtido calculando-se a diferença entre o valor da componente da função objetivo, relativa ao professor, nas configurações nova e antiga, isto é:

$$
\Delta f o_{i}(k, \bar{k})=f o_{i}(\bar{k})-f o_{i}(k) \text {. }
$$

A Tabela 1 mostra um fragmento de um quadro $Q_{1}$ de horários de professores. Cada linha $i$ representa um professor $(i=P 1, P 2, P 3, P 4)$ e cada coluna $k$ um horário $(k=H 1, H 2, H 3, H 4, H 5)$ de um mesmo dia. Cada elemento $(i, k)$ dessa tabela representa a atividade do professor $i$ no horário $k$. A, B, C e D são turmas. Um traço (-) significa indisponibilidade do professor, enquanto uma célula vazia indica que não há atividade no horário. A coluna $f o_{i}$ indica o valor da função objetivo de cada professor, expressa em 2.1, com $\alpha_{i}=1$ e $\beta_{i}=0 \forall i$. Para esse quadro de horários, tem-se $f_{o}\left(Q_{1}\right)=f_{O_{P 1}}+f_{O_{P 2}}+f_{O_{P 3}}+f_{O_{P 4}}=1+2+0+0=3$.

\begin{tabular}{||c||c|c|c|c|c||}
\hline & H1 & H2 & H3 & H4 & H5 \\
\hline P1 & A & & B & B & \\
\hline P2 & B & & & A & A \\
\hline P3 & & B & A & C & B \\
\hline P4 & C & A & C & D & - \\
\hline
\end{tabular}

\begin{tabular}{|c||}
\hline$f o_{i}$ \\
\hline 1 \\
\hline 2 \\
\hline 0 \\
\hline 0 \\
\hline
\end{tabular}

Tabela 1: Quadro $Q_{1}$

A Figura 1 representa $G_{A}$, o grafo da turma A. Cada horário é representado por um vértice, ao qual está associado um professor. $\mathrm{O}$ arco $(H 1, H 5)$ de custo -1 indica que se o prof. $P 1$ mudar sua aula do horário $H 1$ para o horário $H 5$, haverá uma diminuição no valor da função objetivo de 1 unidade $\left(\Delta f_{O_{P}}(H 1, H 5)=\right.$ $\left.f_{P 1}(H 5)-f_{P 1}(H 1)=0-1=-1\right)$.

Uma observação deve ser feita com relação às aulas duplas. De forma a não violar a viabilidade admite-se apenas que uma dessas aulas possa ser transferida de horário e, mesmo assim, para um horário do mesmo dia e de forma tal que a consecutividade das aulas seja preservada. Na Tabela 1 a aula da turma A dos horários $H 4$ e $H 5$ é dupla e, assim, na Figura 1, o único movimento possível, que 


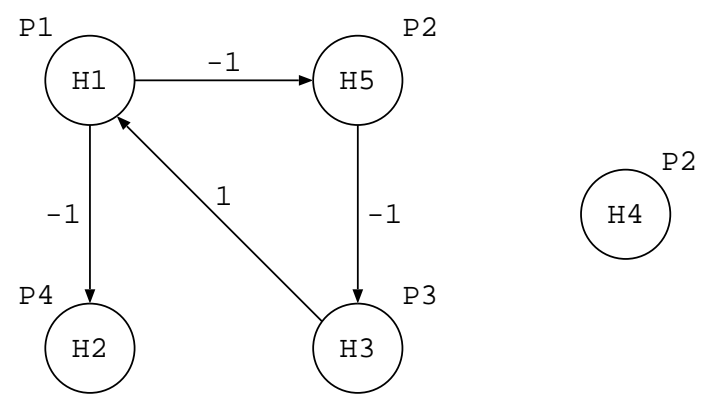

Figura 1: $G_{A}$, Grafo da Turma A

preserva a consecutividade das aulas, é o deslocamento da aula do horário $H 5$ para o horário $H 3$.

Para encontrar um quadro viável de horário com um valor menor para a função objetivo, deve-se procurar um ciclo de custo negativo em $G_{j}$. Observa-se que a existência de um ciclo garante a re-alocação de todas as aulas envolvidas no ciclo e o custo negativo presume uma diminuição de custos.

No exemplo considerado, a sequência de $\operatorname{arcos}\{(H 1, H 5),(H 5, H 3),(H 3, H 1)\}$, forma um ciclo de custo total $-1(=-1+(-1)+1)$. A Tabela 2 apresenta $\mathrm{O}$ quadro $Q_{1}^{\prime}$ de horários da turma $A$ após os movimentos e a Figura 2, o grafo $G_{A}^{\prime}$ correspondente. Para $Q_{1}^{\prime}$ tem-se $f o\left(Q_{1}^{\prime}\right)=0+1+1+0=2$.

\begin{tabular}{||c||c|c|c|c|c||}
\hline & H1 & H2 & H3 & H4 & H5 \\
\hline P1 & & & B & B & A \\
\hline P2 & B & & A & A & \\
\hline P3 & A & B & & C & B \\
\hline P4 & C & A & C & D & - \\
\hline
\end{tabular}

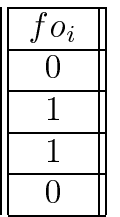

Tabela 2: Quadro $Q_{1}^{\prime}$

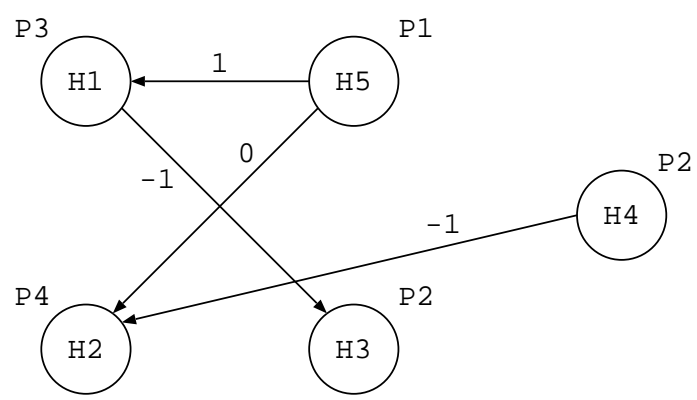

Figura 2: $G_{A}^{\prime}$, Grafo da Turma A após os movimentos candidatos 
Em $G_{A}^{\prime}$ (Figura 2) existem arcos de custo negativo, mas inexistem ciclos de custo negativo envolvendo esses arcos, sinalizando que o quadro de horários da turma $A$ não pode mais ser melhorado com movimentos entre aulas de uma mesma turma (os quais serão chamados movimentos intraturmas). A idéia, então, é repetir o procedimento para uma outra turma e assim sucessivamente até que não seja possível melhorar o quadro de horários dos professores através de movimentos intraturmas.

Chama-se a atenção para o fato de que a existência de um ciclo de custo negativo pode, no entanto, não garantir melhora no valor da função objetivo, assim como pode gerar soluções inviáveis, conforme exemplifica-se a seguir.

A Tabela 3 representa um fragmento de um quadro $Q_{2}$ de horários de professores, de 2 dias com 5 horários cada. A função objetivo é avaliada conforme 2.1 , com $\alpha_{i}=1$ e $\beta_{i}=2 \forall i$ e, no grafo $G_{A}$ (Figura 3 ), existe um ciclo de custo -1 .

\begin{tabular}{||c||c|c|c|c|c||c|c|c|c|c||}
\hline & H1 & H2 & H3 & H4 & H5 & H6 & H7 & H8 & H9 & H10 \\
\hline P1 & A & & A & & & A & & & & \\
\hline P2 & & A & & & & & & B & B & A \\
\hline P3 & & & & A & A & & A & & & \\
\hline P4 & & & & & & & & A & A & \\
\hline
\end{tabular}

\begin{tabular}{|c|}
\hline$f o_{i}$ \\
\hline 5 \\
\hline 4 \\
\hline 4 \\
\hline 2 \\
\hline
\end{tabular}

Tabela 3: Quadro $Q_{2}$

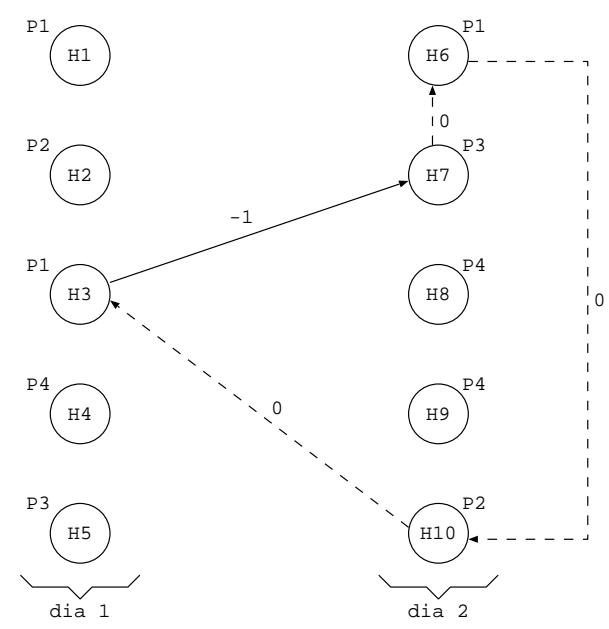

Figura 3: Exemplo de um ciclo de custo negativo que não representa melhora no valor da função objetivo

O quadro $Q_{2}^{\prime}$ resultante (Tabela 4) mostra que, na realidade, o valor da função objetivo aumentou em 1 unidade, ao invés de diminuir de uma unidade, conforme se presumia. 


\begin{tabular}{||c||c|c|c|c|c||c|c|c|c|c||}
\hline & H1 & H2 & H3 & H4 & H5 & H6 & H7 & H8 & H9 & H10 \\
\hline P1 & A & & & & & & A & & & A \\
\hline P2 & & A & A & & & & & B & B & \\
\hline P3 & & & & A & A & A & & & & \\
\hline P4 & & & & & & & & A & A & \\
\hline
\end{tabular}

\begin{tabular}{|c|}
\hline$f o_{i}$ \\
\hline 6 \\
\hline 4 \\
\hline 4 \\
\hline 2 \\
\hline
\end{tabular}

Tabela 4: Quadro $Q_{2}^{\prime}$

Outra situação que pode ocorrer está exemplificada pela Figura 4. Supondo que cada professor possa ministrar no máximo dois horários de aula por dia para cada turma, são possíveis os movimentos das aulas dos horários $H 6$ e $H 11$ para o dia 1, tomados individualmente. No entanto, se os arcos correspondentes a esses movimentos fizerem parte de um mesmo ciclo de custo negativo, tal como o mostrado na Figura 4, a viabilidade não estará garantida.

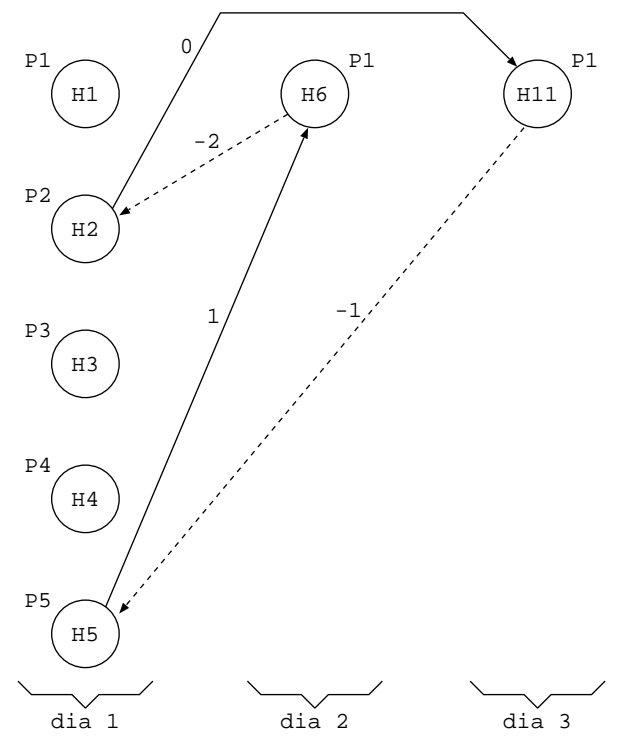

Figura 4: Exemplo de um ciclo no qual um professor, P1, participa em mais de um arco e a viabilidade é violada.

Essas duas últimas situações, no entanto, só ocorrem quando um mesmo professor participa em mais de um arco no ciclo. Assim, faz-se necessário checar a viabilidade e o valor da função objetivo após os movimentos candidatos.

Sugere-se, portanto, um procedimento iterativo que considere, a cada vez, uma turma. Para cada turma $j$ constrói-se seu grafo $G_{j}$ e aplica-se um algoritmo para detectar ciclos de custo negativo. Enquanto houver ciclo de custo negativo que melhore o valor da função objetivo e não produza inviabilidade, realizam-se os movimentos, atualizando-se o quadro de horários, bem como o grafo $G_{j}$. Inexistindo mais tais 
ciclos no grafo da turma $j$ parte-se para uma outra turma. Esse procedimento é encerrado quando nenhum movimento de melhora for possível para todas as turmas. O Algoritmo Intraturmas, descrito na Figura 5, formaliza esse procedimento.

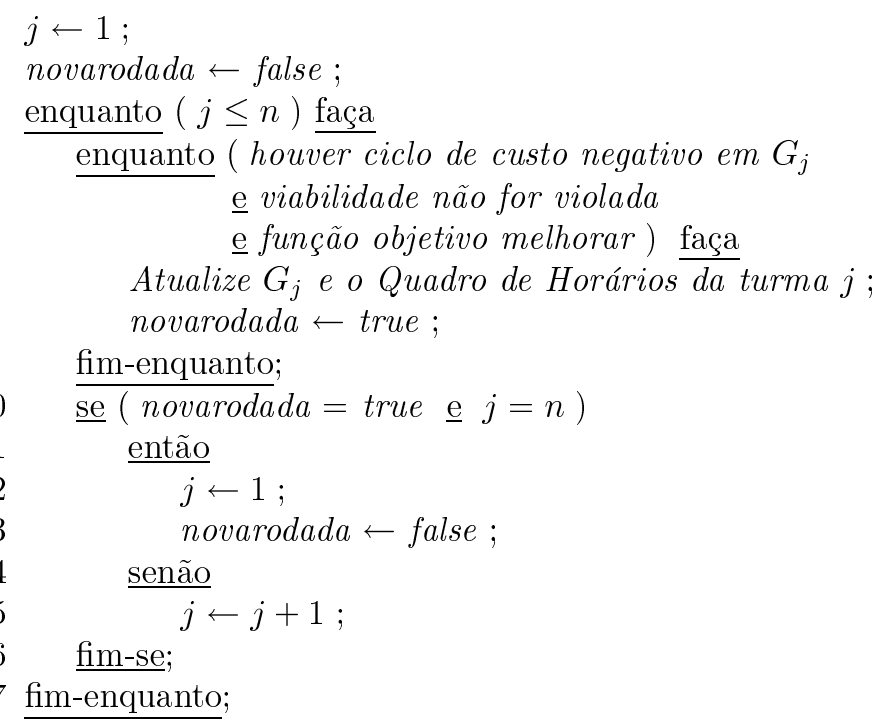

im-enquanto;

Figura 5: Algoritmo Intraturmas

Ao final do Algoritmo Intraturmas podem restar, ainda, arcos de custo negativo nos grafos das turmas. A Figura 6 , que considera os grafos de duas turmas, $j$ e $\bar{j}$, ilustra tal situação.

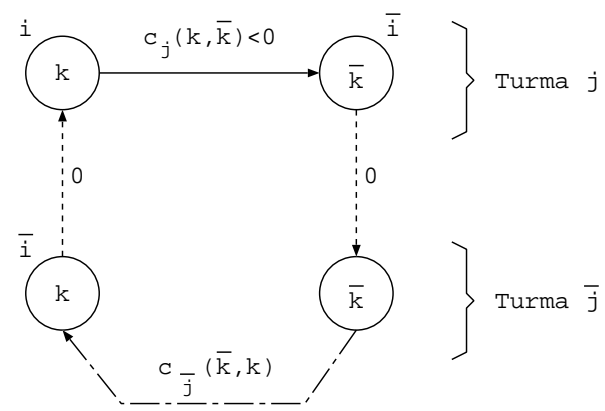

Figura 6: Grafos das turmas $j$ e $\bar{j}$ resultantes do Algoritmo Intraturmas

Nessa figura, há um arco de custo negativo na turma $j$, de $k$ a $\bar{k}$, a saber $c_{j}(k, \bar{k})$, sinalizando que poderá haver uma melhora no valor da função objetivo se o prof. $i$ tiver sua aula do horário $k$ transferida para o horário $\bar{k}$. Entretanto, essa transferência não pode ocorrer porque o professor $\bar{i}$, do horário $\bar{k}$, não está 
disponível no horário $k$ (Nesse horário, ele está ministrando aula para a turma $\bar{j}$ ). Nesse caso, como não há custos envolvidos na transferência de um professor de uma turma para outra em um mesmo horário, e de forma a tentar obter um ciclo de custo negativo, pode-se transferir o professor $\bar{i}$ da turma $j$ para a turma $\bar{j}$ no horário $\bar{k}$ e da turma $\bar{j}$ para a turma $j$ no horário $k$ (arcos de custo nulo da Figura 6, que ligam os grafos das duas turmas) procurando-se, a seguir, em $G_{\bar{j}}$, o caminho mínimo de $\bar{k}$ a $k$, de sorte que $c_{j}(k, \bar{k})+c_{\bar{j}}(\bar{k}, k)<0$, onde $c_{\bar{j}}(\bar{k}, k)$ é o custo do caminho mínimo de $\bar{k}$ a $k$ em $G_{\bar{j}}$.

Assim, para cada arco $(k, \bar{k}) \in G_{j}$, de custo negativo, pesquisa-se a existência de um ciclo de custo negativo envolvendo esse arco de $G_{j}$, o grafo $G_{\bar{j}}$ e os arcos de custo nulo que os conectam. Assim como no Algoritmo Intraturmas, faz-se necessário checar a viabilidade e o valor da função objetivo antes e depois dos movimentos candidatos.

O Algoritmo Interturmas, descrito na Figura 3., formaliza essa idéia.

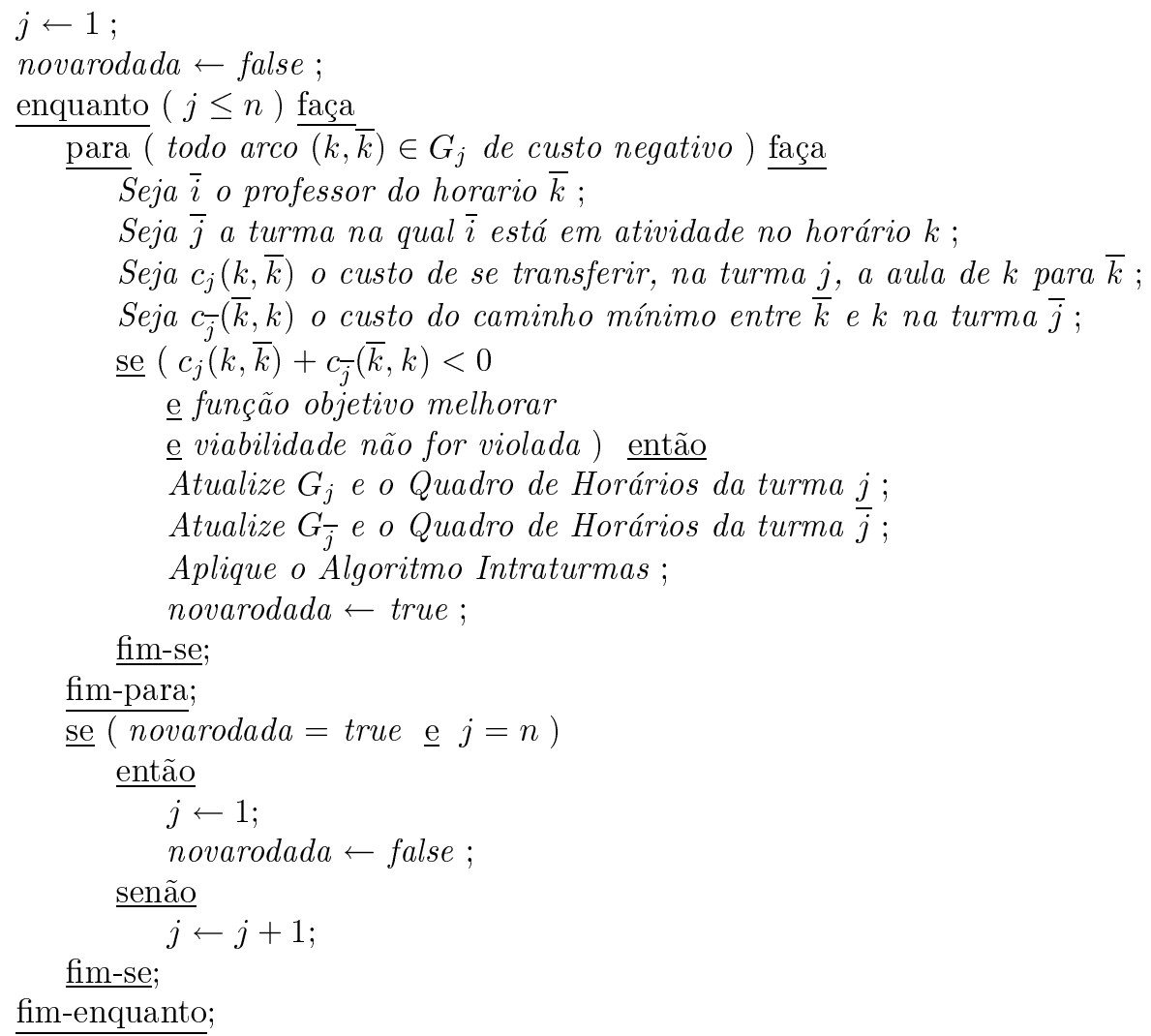

Figura 7: Algoritmo Interturmas

Observa-se que toda vez que o Algoritmo Interturmas produz uma solução melhor, o Algoritmo Intraturmas é novamente acionado, uma vez que o quadro de 
horários dos professores poderá ser melhorado com movimentos intraturmas, em função das alterações promovidas nos quadros de horários das turmas $j$ e $\bar{j}$.

Em resumo, propõe-se a aplicação de um procedimento de duas fases. Na primeira fase, aplica-se o Algoritmo Intraturmas, resultando a existência de $n$ grafos $G_{j}, \forall j=1, \ldots, n, \mathrm{com}$, possivelmente, arcos de custo negativo. Encerrada a primeira fase, aplica-se o Algoritmo Interturmas.

\section{Resultados Computacionais Preliminares}

Apesar de o PHE ser um problema clássico da área de otimização, inexiste um conjunto de problemas-teste que possa ser usado para avaliar a qualidade das soluções geradas pelo procedimento proposto. Isto se deve à diversidade de regimes de ensino e às características específicas de cada instituição de ensino, o que torna praticamente ímpar cada PHE.

Em visto do exposto, o procedimento de duas fases apresentado foi testado utilizando-se 3 problemas-teste, para os quais desconhece-se a solução ótima. Os dois primeiros foram utilizados em [6] e o último refere-se ao planejamento de aulas do turno da manhã do primeiro semestre letivo de 1998 da Escola Estadual Dom Silvério, em Mariana (MG). Para todos os problemas-teste tomou-se $\alpha_{i}=1$ e $\beta_{i}=2$ $\forall i=1, \ldots, m$, priorizando-se, portanto, a redução do número de dias em que cada professor esteve envolvido em atividades de ensino.

O procedimento foi implementado na linguagem $\mathrm{C}$ e testado em uma estação SUN Sparc Ultra 1. Para determinar ciclos de custo negativo implementou-se o Algoritmo de Floyd ([2]). As soluções iniciais, exceto a última do problema 3, que foi elaborada manualmente em 2 semanas de trabalho pelo planejador de horários da instituição, foram geradas pelo otimizador XPRESS/MP, versão 11.02. A Tabela 5 sumariza os resultados computacionais obtidos.

\begin{tabular}{|c|c|c|c|c|c|c|c|}
\hline Prob. & $\mathrm{m}$ & $\mathrm{n}$ & $\begin{array}{c}\text { fo } \\
\text { inicial }\end{array}$ & $\begin{array}{c}\text { fo após } \\
\text { Intraturmas }\end{array}$ & $\begin{array}{c}\text { fo após } \\
\text { Interturmas }\end{array}$ & $\begin{array}{c}\% \text { de } \\
\text { redução }\end{array}$ & $\begin{array}{l}\text { Tempo de } \\
\text { CPU (s) }\end{array}$ \\
\hline \multirow{5}{*}{1} & \multirow{5}{*}{8} & \multirow{5}{*}{3} & 76 & 64 & 64 & 28.9 & 0.07 \\
\hline & & & 72 & 64 & 64 & 25.0 & 0.06 \\
\hline & & & 63 & 59 & 59 & 6.3 & 0.04 \\
\hline & & & 60 & 59 & 59 & 1.7 & 0.02 \\
\hline & & & 57 & 57 & 57 & 0.0 & 0.03 \\
\hline \multirow{3}{*}{2} & \multirow{3}{*}{16} & \multirow{3}{*}{8} & 171 & 124 & 120 & 29.8 & 0.37 \\
\hline & & & 157 & 126 & 118 & 24.8 & 0.60 \\
\hline & & & 131 & 116 & 116 & 11.5 & 0.18 \\
\hline \multirow{3}{*}{3} & \multirow{3}{*}{31} & \multirow{3}{*}{13} & 297 & 199 & 185 & 37.7 & 2.24 \\
\hline & & & 237 & 193 & 187 & 21.1 & 1.05 \\
\hline & & & 211 & 190 & 173 & 18.0 & 1.47 \\
\hline
\end{tabular}

Tabela 5: Resultados computacionais 


\section{Conclusões}

Apresentou-se um procedimento de duas fases que, efetivamente, melhora quadros viáveis de horários para o caso em que o número de horários reservados para as aulas é exatamente igual à carga horária de aulas de cada turma. Tal procedimento navega no espaço das soluções viáveis e pode ser facilmente inserido em metaheurísticas tais como Busca Tabu (na fase de intensificação), GRASP (na fase de busca local) e outras (Simulated Annealing, Algoritmos Genéticos, etc), tão logo uma solução viável esteja disponível. Evidentemente, a técnica proposta pode ser aplicada para tratar também de outras restrições, além daquelas mencionadas na seção 2, tais como: limite diário de aulas por professor, intervalo entre aulas de um mesmo professor para uma mesma turma, etc.

\section{Referências}

[1] D. Abramson, Constructing school timetables using Simulated Annealing: sequential and parallel algorithms, Management Science, 37 (1991), 98-113.

[2] R.K. Ahuja, T.L. Magnanti and J.B. Orlin, "Network flows: theory, algorithms and applications", Prentice Hall, New Jersey, 1993.

[3] A. Colorni, M. Dorigo and V. Maniezzo, Metaheuristcs for high school timetabling, Computational Optimization and Applications, 9 (1998), 275-298.

[4] D. Costa, A tabu search algorithm for computing an operational timetable, European Journal of Operational Research, 76 (1994), 98-110

[5] S. Even, A. Itai and A. Shamir, On the complexity of timetabling and multicommodity flow problems, SIAM Journal of Computation, 5 (1976), 691-703.

[6] S.S. Mata, "O Problema do Horário na Escola de Segundo Grau: Modelagem e Implementação", Tese de mestrado, Progr. de Eng. de Sistemas e Computação, COPPE/UFRJ, Rio de Janeiro, 1989.

[7] A. Schaerf, A survey of automated timetabling, Artificial Intelligence Review, 13 (1999), 87-127.

[8] A. Schaerf, "Tabu search techniques for large high-school timetabling problems", Technical Report, CWI, Amsterdan, 1996.

[9] M.J.F. Souza, "O Problema do Horário em Escolas: uma aproximação por metaheurísticas", Exame de qualificação ao doutorado, Progr. de Eng. de Sistemas e Computação, COPPE/UFRJ, Rio de Janeiro, 1998. 